\title{
Molecular evolution of LysR-type transcriptional regulation in Pseudomonas aeruginosa
}

\author{
F. Jerry Reen, Matthieu Barret, Emilie Fargier, Marcus O’Muinneacháin, Fergal O’Gara* \\ BIOMERIT Research Centre, Department of Microbiology, University College Cork, Cork, Ireland
}

\section{A R T I C L E I N F O}

\section{Article history:}

Received 25 September 2012

Revised 13 December 2012

Accepted 17 December 2012

Available online 27 December 2012

\section{Keywords:}

LysR-Type Transcriptional Regulator

Pseudomonas aeruginosa

Horizontal gene transfer

Phylogenetic analysis

Divergent evolution

\begin{abstract}
A B S T R A C T
Signal perception and transduction through tightly coordinated circuits is integral to the survival and persistence of microbes in diverse ecological niches. The capacity to adapt to changes in the environment is central to their ability to thrive under adverse circumstances. Signal dependent transcriptional regulators are a key mechanism through which microbes assimilate environmental cues and mediate the appropriate adaptive response. By far the largest class of transcriptional regulator is the LysR-class, which is universally distributed among bacteria, archaea, and even eukaryotic organisms. The number of LysRType Transcriptional Regulators (LTTRs) varies among species with one of the largest repertoires encoded in the genome of the nosocomial pathogen Pseudomonas aeruginosa. To understand the evolutionary basis for this, we undertook to analyse the relationship between the LTTRs, both at the species and genus level. Phylogenetic analysis of the complete Pseudomonas LTTR dataset revealed significant cluster patterns based on full length and domain analysis. Interestingly, evidence of acquisition through horizontal gene transfer was rare, with divergent evolution apparently favoured. Furthermore, genes that appear to have been acquired, as well as those with a non-classical topological arrangement were clustered in distinct groups in the phylogenetic trees, indicating some ancestral association. The conservation within clusters identified in this study will provide a useful platform for future molecular analyses.
\end{abstract}

(c) 2012 Elsevier Inc. All rights reserved.

\section{Introduction}

Microbial adaptation and niche persistence is facilitated by the exquisite coordination of cellular function at the level of transcription, post-transcription and metabolic control. The ability to adapt to changes in the environment or to avoid predation from higher organisms is a key component of their ability to thrive under adverse circumstances. This requires the action of DNA-binding transcriptional regulators, post-transcriptional regulators and posttranslational modification, and metabolic control by enzymes. While signal perception and response is often considered to be associated with membrane proteins, and in particular with two component sensors, transcriptional regulators are increasingly been identified as signal dependent regulators of bacterial adaptation. By far the largest class of transcriptional regulator is the LysR-class, which is universal among both Gram-negative and Gram-positive bacteria. Since LysR-type Transcriptional Regulators (LTTRs) were first classified as a distinct regulatory family (Henikoff et al., 1988), the number of LTTRs identified has grown from 9 to over 40,000 (Krushkal et al., 2012; Maddocks and Oyston, 2008; Monferrer et al., 2010; Schell, 1993). Capable of mediating

\footnotetext{
* Corresponding author. Fax: +353 214275934.

E-mail address: f.ogara@ucc.ie (F. O’Gara).
}

both signal dependent and signal-independent transcriptional regulation, LTTR orthologues are also found in archaea and eukaryotic organisms (Maddocks and Oyston, 2008).

LTTRs have been found to control a diverse range of cellular functions, such as virulence factor expression, stress response, motility, quorum sensing, antibiotic resistance, fixation of $\mathrm{CO}_{2}^{-}$ and $\mathrm{N}_{2}^{-}$, biosynthesis of amino acids and catabolism of aromatic compounds among other activities (Maddocks and Oyston, 2008; Tropel and van der Meer, 2004). LTTRs function by activating or repressing the divergent transcription of target genes. Divergent transcription is believed to have advantages over non-divergent transcription (Beck and Warren, 1988). The advantage of this arrangement is that a single transcriptional regulatory protein can regulate two sets of genes, minimising the need for multiple regulatory proteins. However, crucially from the perspective of evolution, divergently transcribed transcriptional units are believed to be more stable during recombination and genetic rearrangement. This is due to the fact these independent units are maintained upon translocation as the promoters of divergent gene units are internal (Beck and Warren, 1988).

Pseudomonads occupy a diverse spectrum of ecological niches and have quite diverse and varied lifestyles. Several Pseudomonas species have been widely studied as beneficial factories for plant development and bioremediation, while other species are 
considered pathogenic to plants and animals. This capacity for broad spectrum niche occupancy may be attributed in part to the vast repertoire of regulatory proteins encoded in the Pseudomonas genome. In the important nosocomial pathogen Pseudomonas aeruginosa, LTTRs such as MexT and PqsR have emerged as key modulators of cell-cell signalling, virulence, antibiotic resistance and pathogenesis (Deziel et al., 2005; Tian et al., 2009a,b). The capacity for this important pathogen to adapt and persist in diverse ecological niches underpins its position as one of the top three nosocomial pathogens and the primary pathogen associated with cystic fibrosis (CF) patients (Govan and Deretic, 1996). The mechanism of action of LTTR proteins is largely conserved and there is a strong likelihood that significant clues as to the functionality of these proteins may be gleaned from sequence conservation and understanding the evolutionary mechanisms underlying the emergence of the large abundance of this class of transcriptional regulator in P. aeruginosa and other Gram negative pathogens. Therefore we performed phylogenetic and cluster analyses of the Pseudomonas LTTR repertoire with a view towards understanding the relationship between these key regulators of microbial behaviour. This may provide a scaffold or platform for the future comprehensive characterisation of LTTR regulation in this bacterial genus.

\section{Materials and methods}

\subsection{Analysis of the LTTRs distribution in genome sequences}

The following protein families COG0583, Pfam0126 and Pfam03466 were used to investigate the occurrence of LTTRs in bacterial genome sequences available in the Integrated Microbial Genomes (IMG) database at the time of writing (Markowitz et al., 2012). The average frequency of LysR per Mbp and the associated standard deviation was calculated for each bacterial phylum and proteobacterial class as well as for some gamma-proteobacterial genera.

\subsection{Recovery of Pseudomonas LysR regulator sequences}

LysR sequences were retrieved from all the Pseudomonas genomes present the IMG database at the time of analysis (Table S1) (Markowitz et al., 2012). The following conserved domain accession number COG0583, Pfam0126 and Pfam03466 were applied to identify LysR homologues. Incomplete LysR amino acid sequences (less than 250 amino acids) were removed and 112 LysR homologue annotated in the P. fluorescens F113 genome (Redondo-Nieto et al., 2012) were added to the Pseudomonas LysR database. All the LysR proteins (3853) were clustered using BLASTclust with a very high sequence identity threshold (an identity of $80 \%$ in an alignment which covers at least $90 \%$ of both sequences). One amino acid sequence representative of each cluster (767) was chosen and these proteins were aligned using the default parameter of MAFFT (Katoh and Toh, 2008). Based on this alignment two proteins were discarded (PSPPH_3278 and PA2492) as they present two unique insertions sites, which are probably the result of mis-annotation.

\subsection{Phylogenomics/phylogenetic analyses}

Pseudomonas aeruginosa predicted proteomes were downloaded from the NCBI ftp server (Table S1). Whole-genome based phylogenetic trees were built by using a composition vector approach ( $\mathrm{Li}$ et al., 2010; Qi et al., 2004) using the web server CVTree (http:// tlife.fundan.edu.cn/cvtree/) with a peptide window ( $k$ value) equal to 6 . Trees were generated by Neighbour joining algorithm using as outgroup Escherichia coli K12-DH10B. Maximum-Likelihood (ML) trees were built with PhyML (Guindon and Gascuel, 2003) using the WAG amino acid substitution model of evolution (Whelan and Goldman, 2001) and four categories of substitution rates. Branch supports were evaluated using the approximate likelihood-ratio test (aLRT) (Anisimova and Gascuel, 2006).

A binary matrix was constructed on the basis of the presence/ absence of LTTRs in P. aeruginosa strains. From this matrix a dendrogram was constructed using Euclidean distance and Neighbour-Joining method. All Phylogenetic trees were visualised and exported using the web-based tool Interactive Tree Of Life (Letunic and Bork, 2011).

\subsection{Comparative analysis of LTTRs and other TR families in $P$. aeruginosa}

The relative abundance of LTTRs among the 500 predicted transcriptional regulators annotated in $P$. aeruginosa genomes, was assessed by COG prevalence (http://www.ncbi.nlm.nih.gov/COG/). The top 10 families/groups of transcriptional regulators (functional category of transcriptional regulators) were tabulated and displayed in pie-chart format using R statistical analysis software.

\subsection{Construction of the Pseudomonas aeruginosa LTTR full length and domain trees}

LTTR protein sequences were obtained from $P$. aeruginosa strains PA14. LTTRs that were absent from PA14 (on the basis of the previous clustering), but present in the other $P$. aeruginosa genomes used in this study were also included in the analysis. All sequences were aligned with MAFFT and phylogenetic analysis was performed as described in section phylogenetic analyses. In addition to phylogenetic analysis of the full LTTR sequences ( $\sim 300$ residues), the $\mathrm{N}$-terminus (first 70 residues) and the C-terminus (last 200 residues) domains of the protein were also phylogenetically analysed. To aid in the visual assessment of congruency or incongruence between the full LTTR, DNA-binding Domain (DBD) and Co-inducer Binding Domain (CBD) trees, conserved domains (CDs) were overlaid on the three phylogenetic trees (detailed in Table S2). Minimum bootstrap support values $\geqslant 50 \%$ were enforced for this analysis.

\subsection{Analysis of horizontal acquisition and topological arrangement}

The complete repertoire of $P$. aeruginosa LTTRs were analysed for compositional bias of dinucleotide frequency (GC skew) using the online program deltarho (http://deltarho.amc.uva.nl) (van Passel et al., 2005). The program calculates the average dinucleotide relative abundance difference (genomic dissimilarity value, $\delta^{*}$ ) between input sequences (LTTR nucleotide sequences) and the relevant $P$. aeruginosa genome sequence (van Passel et al., 2005). A high genomic dissimilarity value indicates that the input sequence may be of heterologous origin and in this study a genomic dissimilarity value exceeding 65 was considered a HGT candidate, and was further investigated using WebACT (http://www.webact.org) (Abbott et al., 2005). Divergence was investigated using DnaSP v5.10.01 software (Rozas et al., 2003). The standard McDonaldKreitman test was performed on full length LysR protein sequences which were present in all $P$. aeruginosa strains and also in $P$. fluorescens, P. putida, and P. syringae. Four housekeeping genes (dnaK, $r p o D$, gyrB, and recA) were also selected for analysis based on previous studies (Bennasar et al., 2010). The neutrality index (NI) was calculated based on the ratio of polymorphisms to substitutions as follows: $\mathrm{NI}=(\mathrm{Pn} / \mathrm{Ps}) /(\mathrm{Dn} / \mathrm{Ds})$, where $\mathrm{P}$ signifies polymorphism within the species and $\mathrm{D}$ represents divergence between species. P. aeruginosa strains PA14, PAO1, PA7, PACS2, 2192, C3719, LESB58, 39016. P. fluorescens SBW25 was chosen as a non-species outlier 
for this analysis. Topological arrangement was investigated using the Pseudomonas database (Winsor et al., 2011). Transcriptional units were classified as divergently transcribed or non-divergently transcribed depending on the arrangement between the LTTR and adjacent genes.

\subsection{Meta-analysis of transcriptomic datasets}

All $P$. aeruginosa transcriptome datasets publicly available were retrieved from the GEO database (http://www.ncbi.nlm.nih.gov/ geo/) when available, or directly from publications. Genes were clustered according to their expression profiles using the MultiExperiment Viewer Software v4.5.1 (http://www.tm4.org/mev/). Genes were grouped either with Pearson correlation or Euclidian distance as distance for $K$-means clustering. The optimal number of expression clusters was chosen after a figure-of-merit algorithm.

\section{Results and discussion}

\subsection{Prevalence of LysR among the Pseudomonadaceae}

LTTRs exist across a diverse range of prokaryotes, although the majority are found in Proteobacteria, especially Gamma and Alpha Proteobacteria, with fewer in the Beta class (Maddocks and Oyston, 2008; Schell, 1993). Significantly fewer LTTRs are found among Gram positive bacteria, however this apparent uneven distribution of LTTRs among bacterial phyla likely reflects the uneven distribution of genetically characterised members of the various subdivisions (Maddocks and Oyston, 2008). The following conserved domain accession numbers COG0583, Pfam0126 and Pfam03466 were applied to investigate the occurrence of LTTRs in bacterial genome sequences available in the IMG database at the time of analysis. LTTRs were found to be almost ubiquitous in every bacterial phylum, with the exception of Dictyoglomi, Tenericutes and Thermotogae. However, the relative abundance of LTTR per genome varies greatly between phyla, ranging from an average of 0.07 LTTR per $\mathrm{Mb}$ for Chlamydia to an average of 7.56 for Proteobacteria (Fig. 1A). LTTRs are also unevenly distributed between Proteobacterial classes (Fig. 1B) and Proteobacterial genera (Fig. 1C). Within the Gamma Proteobacteria, LTTRs are most prevalent in Pseudomonas, Acinetobacter, and Vibrio, while Haemophilus and Xanthomonas exhibit a significantly lower frequency of LTTRs (Fig. 1C).

Previous analysis of LTTR DNA and amino acid sequences has identified significant structural and functional homology, indicating a common evolutionary history (Schell, 1993; Schlaman et al., 1992). It has been proposed that the precursor LTTR arose early in the evolution of prokaryotes, based primarily on the significant genetic distances, distinct \%GC profiles, and the diverse structures of the coinducers needed to activate or repress transcription (Schell, 1993). Schlaman et al. (1992) reported three putative LTTR subgroups $(n=43)$ with no apparent common functions (i.e., different target genes, coinducers, and origin) among the LTTRs (Schlaman et al., 1992). More recently, phylogenetic analysis of the
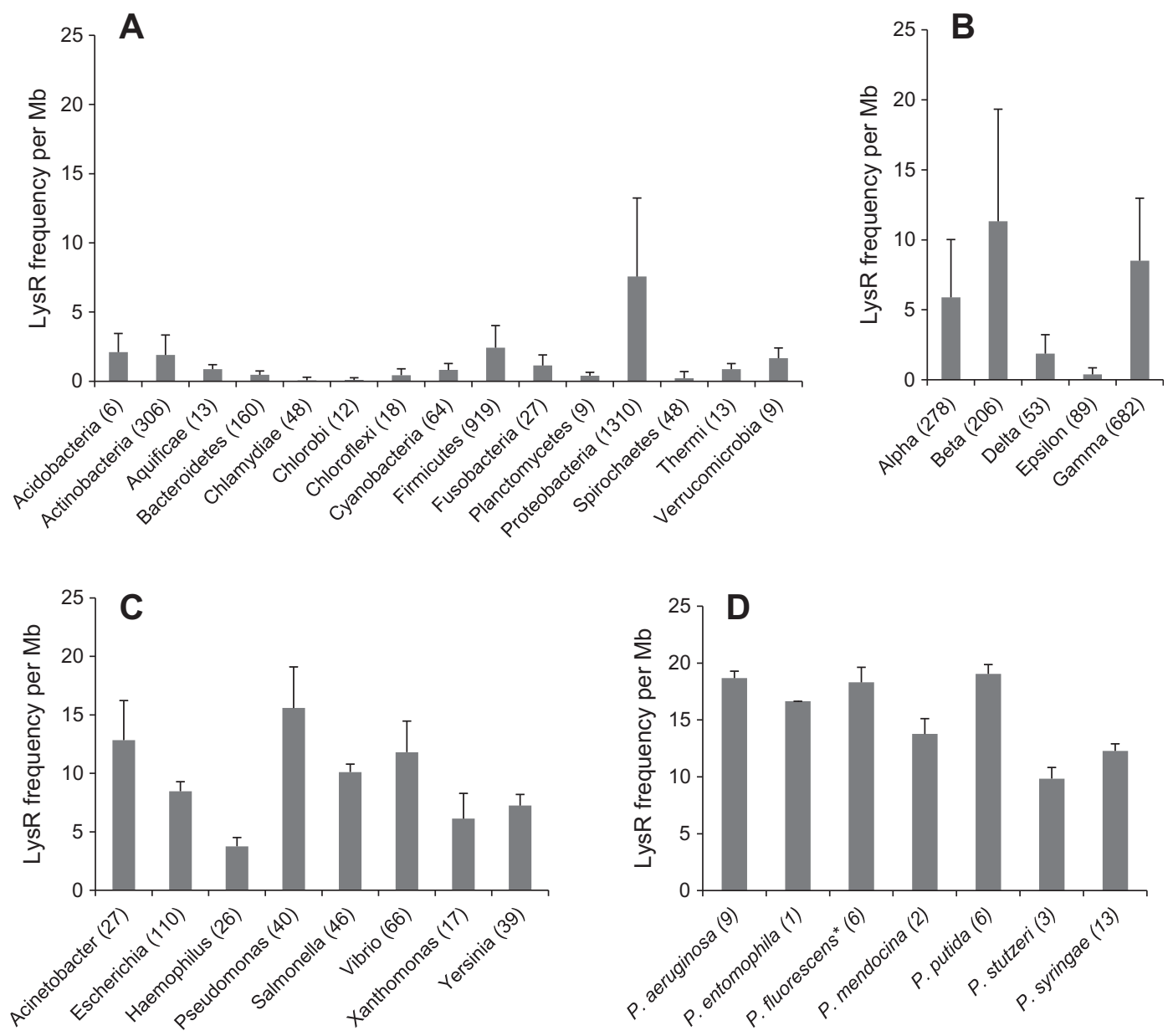

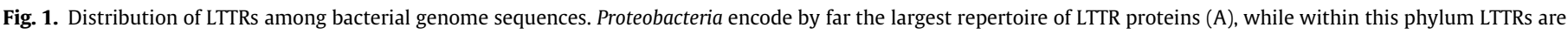

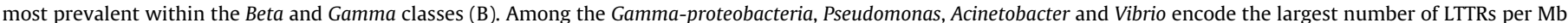

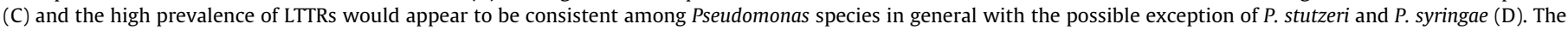
number of genomes employed in each analysis is provided in parentheses. 
Delta-proteobacteria revealed five distinct genetic lineages from the eight Geobacter sulfurreducens LTTR protein sequences within the Geobacteraceae (Krushkal et al., 2012). However, reports at the genus and species level are lacking and therefore we selected Pseudomonads, and in particular the nosocomial pathogen $P$. aeruginosa as a model organism for analysis and characterisation. Interestingly, the frequency of LTTRs per Mb is higher in Pseudomonas than in all the others genera of Gammaproteobacteria (Fig. 1C). Within this genus, the human and plant pathogenic species $P$. aeruginosa and $P$. fluorescens species encode the highest frequency of LTTRs, while the lowest frequency was identified in P. syringae (Table S1 and Fig. 1D). Therefore we decided to investigate in more depth the distribution and sequence relationships between LTTR proteins in this bacterial genus.

\subsection{Phylogenetic analysis of LTTR proteins among Pseudomonads}

To dissect the relationship between the LTTR proteins among Pseudomonads, phylogenetic analysis of 3853 LTTRs from 40 available Pseudomonas genomes (Table S1) was performed. Following this analysis, LTTRs were grouped into nine major phylogenetic clusters based on the tree topology (Fig. 2). Species specific signa-

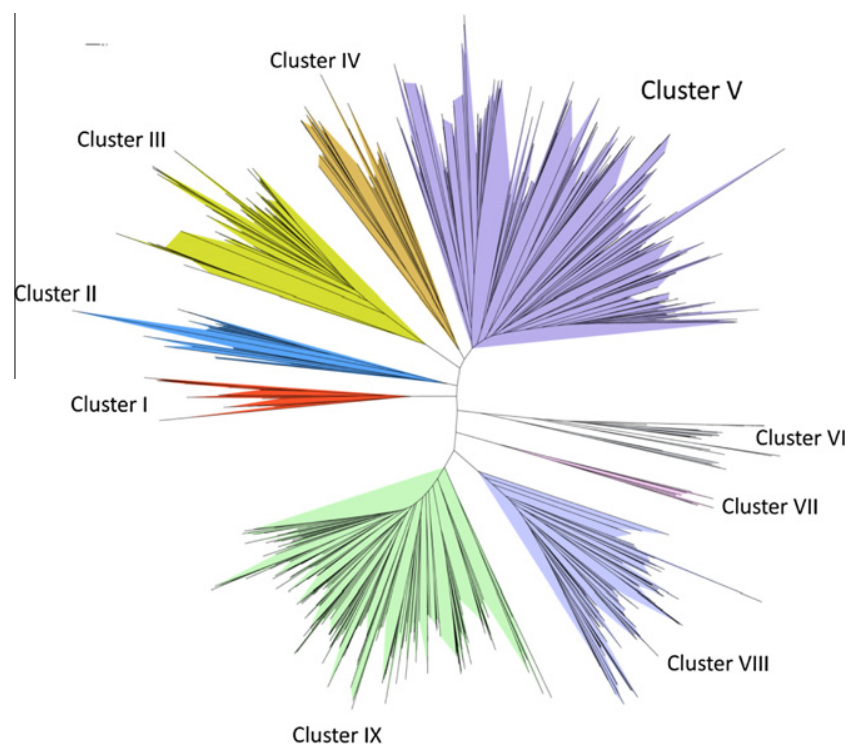

Fig. 2. Phylogenetic analysis of LTTR proteins within the Pseudomonas genus. A distance tree (Maximum-Likelihood) was calculated from 765 Pseudomonas LTTR protein sequences representative of each protein group. Nine main clusters were assigned and each is identified by distinct colour. Clusters V and IX represented by far the largest repertoire of LTTR proteins in the genus, while cluster VII contained the least number of LTTR proteins. Minimum bootstrap support values $\geqslant 50 \%$ were enforced for this analysis. tures were identified in the prevalence of LTTRs within each cluster, with the greatest biased towards cluster $\mathrm{V}$ and cluster IX among all species (Table 1 ).

With the exception of the $P$. aeruginosa virulence regulator PqsR, the LTTRs that form part of cluster I remain uncharacterised. PqsR is a key component of the alkylquinolone QS system in $P$. aeruginosa and its co-inducing signals have been identified as HHQ and PQS (Deziel et al., 2005). The pqsR gene has no orthologues outside of the $P$. aeruginosa genus indicating this key regulator is species specific and evolved after the species diverged from a common ancestor.

Cluster II contains a significant number of LTTRs involved in the regulation of energy metabolism, including homologues of the GbuR and GpuR transcriptional regulators. These have previously been shown to have derived from a common ancestor to control the metabolism of arginine compounds 3-guanidinopropionate and 4-guanidinobutyrate, respectively (Nakada and Itoh, 2005). Their presence in a single cluster provides proof of concept for a functional relationship within the phylogenetic clusters presented in this study. This cluster also contains another LTTR involved in arginine metabolism, BauR, which is specifically involved in utilisation of polyamines. Both GpuR and BauR have no orthologues outside of the $P$. aeruginosa species indicating that these LTTRs have evolved after speciation, while GbuR has 20 orthologues outside of the $P$. aeruginosa species, indicating this LTTR evolved prior to the species divergence from a Pseudomonas common ancestor (Nakada and Itoh, 2005).

Cluster III includes a large number of MexT homologous proteins as well as a NodD LTTR unique to P. fluorescens SBW25. The MexT regulon has recently been elucidated and shown that its binding to a NodD-type box upstream of target promoters leads it to exert a global influence on virulence and pathogenesis in $P$. aeruginosa (Tian et al., 2009a).

Cluster IV contains the LysR LTTR, not present in any $P$. aeruginosa strains, which controls metabolism of diaminopimelate. Orthologues exist in a number of species, including strains of $P$. fluorescens and P. putida. While several P. aeruginosa LTTRs are contained in this cluster, they are currently uncharacterised.

Cluster $\mathbf{V}$ constitutes by far the largest cluster in this analysis (35\% of all the LTTRs) and also encodes the majority of characterised LTTR proteins. These include CysB involved in sulphur metabolism, OxyR the master regulator of the oxidative stress response, CatR which controls benzoate degradation, MetR which is involved in methionine biosynthesis, and CynR and PcaQ which are involved in cyanate and protocatechuate metabolism respectively.

Cluster VI are uncharacterised to date while cluster VII contains the iciA chromosome initiation LTTR. Cluster VIII contains the TrpI, GcvA, BetR and AmpR proteins which are involved in tryptophan metabolism, glycine cleavage, choline metabolism, and beta lactamase activity, respectively. As observed in cluster $\mathrm{V}$, the cluster arrangement of GcvA with AmpR is interesting as GcvA has been

Table 1

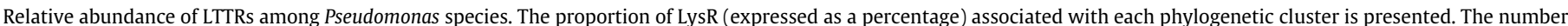
of genomes included in the study are indicated in parentheses.

\begin{tabular}{|c|c|c|c|c|c|c|c|}
\hline Cluster & P. aeruginosa (9) & P.entomophila (1) & P. fluorescens (6) & P. mendocina (2) & P. putida (6) & P. stutzeri (3) & P. syringae (13) \\
\hline I & 3.3 & 2.1 & 2.8 & 3.1 & 4.1 & 2.7 & 1.2 \\
\hline II & 7.3 & 7.2 & 6.2 & 10.8 & 6.7 & 8.1 & 7.3 \\
\hline III & 8.8 & 8.2 & 9.4 & 12.3 & 8.7 & 10.8 & 6.0 \\
\hline IV & 3.4 & 3.1 & 4.4 & 1.5 & 3.9 & 2.7 & 3.3 \\
\hline $\mathrm{V}$ & 35.2 & 35.1 & 33.1 & 29.2 & 36.7 & 32.4 & 33.5 \\
\hline VI & 2.5 & 4.1 & 4.3 & 3.1 & 5.3 & 2.7 & 4.1 \\
\hline VII & 1.6 & 2.1 & 1.2 & 1.5 & 1.8 & 5.4 & 1.4 \\
\hline VIII & 9.9 & 14.4 & 12.6 & 7.7 & 13.7 & 10.8 & 14.5 \\
\hline IX & 28.1 & 23.7 & 25.8 & 30.8 & 19.1 & 24.3 & 28.7 \\
\hline
\end{tabular}


shown to bind AmpR targets in E. coli, mimicking its activated state (Everett et al., 1995).

Finally, cluster IX contains the PtxR LTTR which regulates production of exotoxin A (Ferrell et al., 2008) and PltR, which regulates pyoluteorin production (Yan et al., 2007). Also present in this cluster is the BexR (Bistable expression regulator) of $P$. aeruginosa which is unusual to date in that it functions as a positively regulated bistable epigenetic switch (Turner et al., 2009).

The cluster arrangements observed across the Pseudomonas genus raised important questions as to the relationship between commonly clustered LTTRs and the evolutionary history of their emergence. In particular, the cross-binding of LysR boxes observed to date suggested that at least some of the cluster bias might arise from the highly conserved DNA binding domain (Imperi et al., 2010; Knapp and Hu, 2010; Reen et al., in press; Wei et al., 2012). To address this, we focused on the $P$. aeruginosa genome, in light of the clinical importance of this species and the fact that it contains the largest number of characterised LTTRs.

\subsection{Phylogenetic LTTR domain analysis in P. aeruginosa}

LTTRs have been shown to play key roles in virulence, pathogenesis, and management of a range of stress responses in P. aeruginosa and are by far the largest family of transcriptional regulator encoded in the P. aeruginosa genome (Fig. S1). Therefore, understanding the relationship between LysR proteins in this important pathogen could provide valuable insights into transcriptional control underpinning environmental and niche adaptation. Phylogenetic analysis of the complete LTTR dataset from $P$. aeruginosa PA14 was overall congruent with the Pseudomonas LTTR tree (Fig. S2). However, cluster I and II were not supported as a single clade in the $P$. aeruginosa tree.

LTTRs contain both a DNA Binding Domain (DBD) and Co-inducer Binding Domain (CBD). It is interesting to note that, although LTTR proteins have been shown to contain HTH-domains at the C-terminal (Maddocks and Oyston, 2008), no such domain arrangement was observed in $P$. aeruginosa. As the evolutionary fate of transcription factors is dependent on both the DBD and CBD (Lozada-Chavez et al., 2008), we then decided to perform phylogenetic analysis of the individual domains (Fig. 3).

Conservation of CDs was striking across all phylogenetic trees. CrgA proteins were arranged in two adjacent yet separate clusters (IX and VI), in agreement with the study of Sainsbury and colleagues who reported that $\operatorname{CrgA}$, which assembles into an octameric ring-like structure in contrast with the generally accepted view that LTTRs assemble as homo-tetramers, forms a subclass of LTTRs (Sainsbury et al., 2009). Although fewer in number, LTTRs annotated as being involved in sulphur metabolism (CysL-like), protocatechuate metabolism and aminoethylphosphonate catabolism also exhibit conserved cluster patterns in all three trees (Fig. 3). Furthermore, the AmpR CD was tightly conserved and represented cluster VIII, while the largest cluster $(\mathbf{V})$ contained the greatest diversity of $C D$ as well as the highest proportion of LTTRs for which no domain has been assigned. Within this cluster, the MexT LTTR formed part of a strong clade of 13 proteins. These are largely uncharacterised with only two (MexT and PA2551) LTTRs encoded in all Pseudomonas species. Indeed, the distribution of MexT among Pseudomonads is notable for the degree to which paralogues exist in several species (Table S2). While the significance of this remains to be ascertained through functional genomics approaches, the capacity for MexT to govern virulence and pathogenesis in $P$. aeruginosa suggests this may be highly significant.

Interestingly, the six LTTRs that did not form part of a cluster in this analysis were unique to the $P$. aeruginosa species, a correlation that suggests that these LTTRs are not conducive to evolving for structural or functional reasons. The fact that BexR is one of the few non-clustering LTTR provides a possible insight into why such a small group of LTTRs do not cluster with any other LTTRs in the genome. BexR controls a bistable epigenetic switch that enables the organism to introduce a phenotypically heterogenous subset of cells within an isogenic population, resulting in a group of cells that can survive in an environment that would otherwise have proven fatal (e.g., persistence in the presence of a certain antibiotic) (Turner et al., 2009). The bexR gene is bistably expressed in a BexR-dependent fashion, and its own expression is positively regulated by BexR (Turner et al., 2009). This is novel regulatory mechanism has not yet been attributed to any other characterised LTTR and may explain the lack of close homologues of this and the other unique LTTRs in $P$. aeruginosa.

\subsection{Distribution and topology of LTTR proteins in P. aeruginosa}

The distribution of LTTR proteins was investigated among $P$. aeruginosa sequenced genomes and a significant level of conservation was observed. Approximately 65\% of LTTRs are found in all $P$. aeruginosa isolates (35\% are absent in one or more species), while the number of LTTRs unique to a particular strain is less than $5 \%$ with the exception of PA7 (Fig. 4 and Table S3). Unique LTTRs were distributed among the clusters, although there was a strong bias towards clusters V and IX (Table S3). In many cases, LTTRs were found to be distributed among a subset of strains with 39016 and Pab1 being closest to PA14 in that regard. PA7 has been characterised as a taxonomic outlier, with an unusually broad spectrum of antibiotic resistance, in which key virulence determinants are absent (Roy et al., 2010). In addition to encoding a larger number of distinct LTTRs, PA7 is also notable for the presence of a frameshift mutation of the multiple virulence factor regulator PqsR, which may provide a context for the reduced virulence arsenal of this strain (Roy et al., 2010).

The classical mechanism of LTTR action is regulation of a divergently transcribed promoter in response to activation by co-inducing signal. However, recent reports have highlighted the regulation of distantly transcribed promoters and promoters arranged as part of operon units with the respective LTTR (Krushkal et al., 2012; Maddocks and Oyston, 2008; Tian et al., 2009a). Therefore, the topology of LTTRs in $P$. aeruginosa was investigated using the PA14 strain as baseline. Of the 123 LTTR genes in this strain, 105 were arranged divergent with an adjacent promoter, while only 18 genes encoding LTTRs were arranged in a non-divergent manner (Fig. S3). Interestingly, many of the LTTRs that exhibit a nondivergent topological arrangement are adjacent to each other in binomial or trinomial clusters in the phylogenetic tree. While the rationale underpinning this arrangement is not yet clear, it may indicate divergent evolution of the LTTR and target genes as conserved units. An alternative explanation for this arrangement could be offered were horizontal gene transfer (HGT) to play a significant role in the acquisition of these LTTRs. To determine the extent to which HGT is responsible for the presence and absence of 'unique' LTTRs among $P$. aeruginosa strains, we undertook in silico \%GC content, dinucleotide frequency and genomic dissimilarity analysis.

\subsection{Recent horizontal acquisition of LTTRs is rare in P. aeruginosa}

The expansion of protein families is associated with the acquisition of novel functions and novel regulatory structures essential for the organism to evolve and adapt to new environmental circumstances (Wagner, 2008). Gene duplication is an abundant resource by which genes are created leading to protein family expansion (Wagner, 2008). In this evolutionary mechanism, genes are identical after duplication and functional constraints and natural selection are relaxed to provide opportunities for the gene to evolve, however, if there is ultimately no direct selection on multi- 

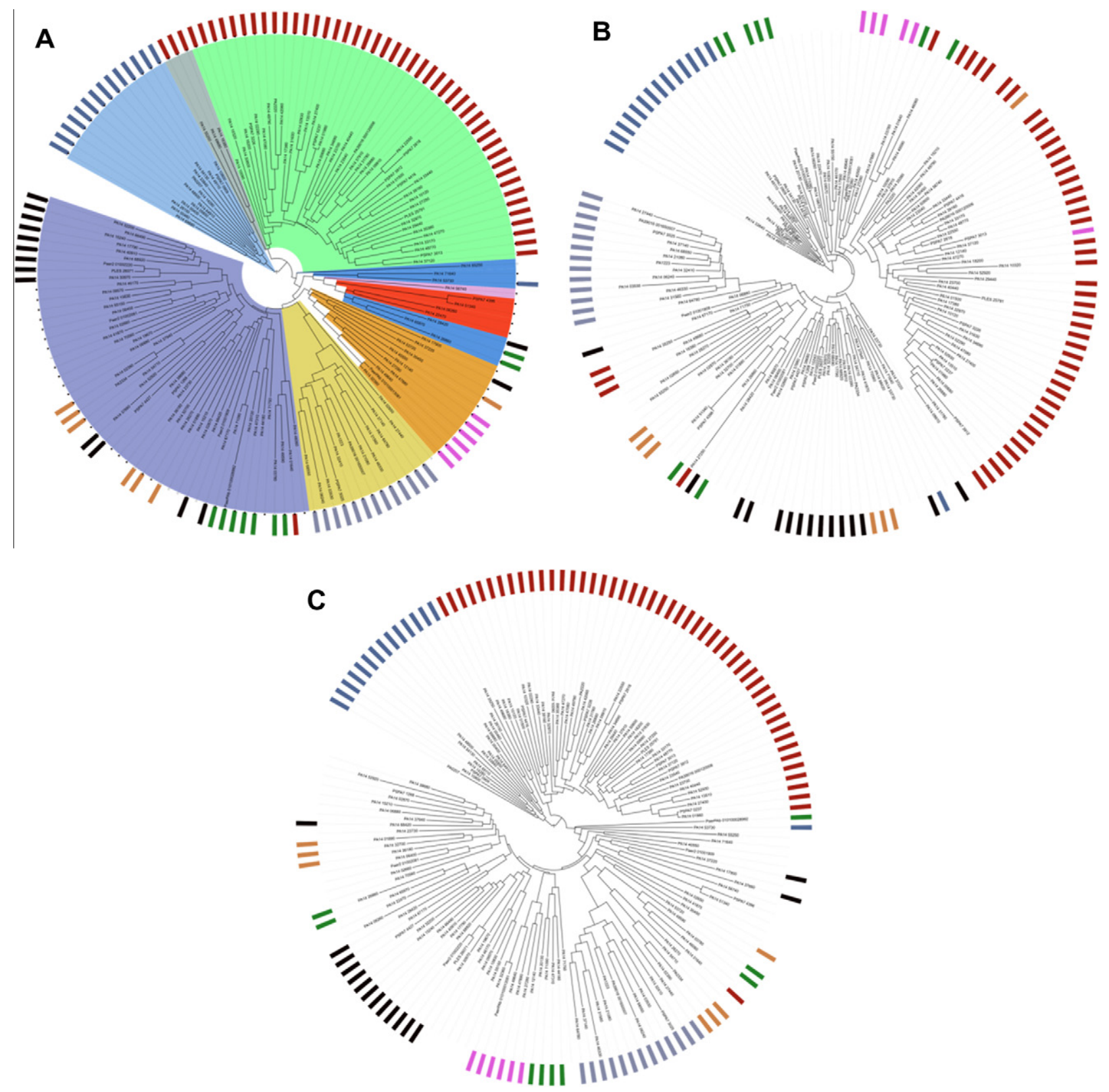

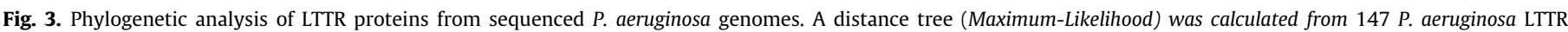

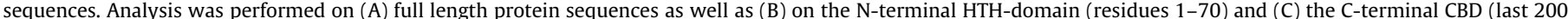

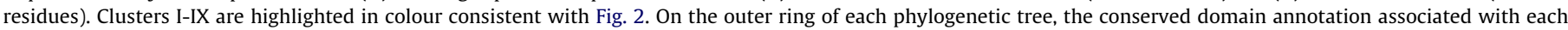

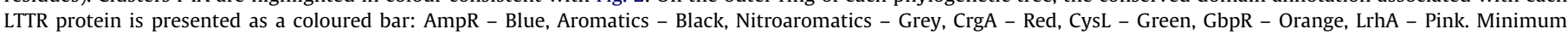

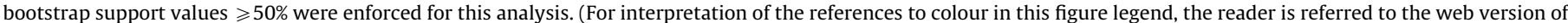
this article.)

ple copies, the redundant extra copies tend to be quickly lost. Such tinkering with pre-existing genes is bypassed in the case of acquisition by HGT (Gogarten et al., 2002). The acquisition of heterologous DNA by means of conjugation, transformation, and transduction can lead to rapid bacterial evolution to adapt to a variety of new niches (Ochman et al., 2000; Pallen and Wren, 2007).

To investigate the contribution of horizontal acquisition to the emergence of LTTRs as the dominant family of transcriptional regulators, LTTR sequences were analysed for comparison with the $P$. aeruginosa PA14 genomic signature using $\delta \rho$-Web (http://deltarho.amc.nl). For robustness, $3 \mathrm{~kb}$ sequences were utilised centred on the respective LTTR. In total, five LTTRs, $b p h R, c y n R$,
PA14_03530, PA14_46330, and PA14_32700, were found to have high genomic dissimilarity $\left(\delta^{*}>65\right)$ and \%GC content relative to PA14 (Table S4). While the insertion site of the horizontally acquired LTTRs has yet to be established, BphR was interesting in this regard. Analysis of the adjacent genes revealed the presence of a prophage region containing genes involved in mobilisation and plasmid transfer/conjugation. To our knowledge, this could be the first evidence for potential plasmid acquisition of LTTRs and further analysis may provide a key insight into the mechanism through which bacteria can incorporate exogenous transcriptional regulatory proteins into their own complex machinery. The BphR LTTR was only found in PA14, 39016 and PA7, while the putative prophage was largely lost from other $P$. aeruginosa strains. Among 


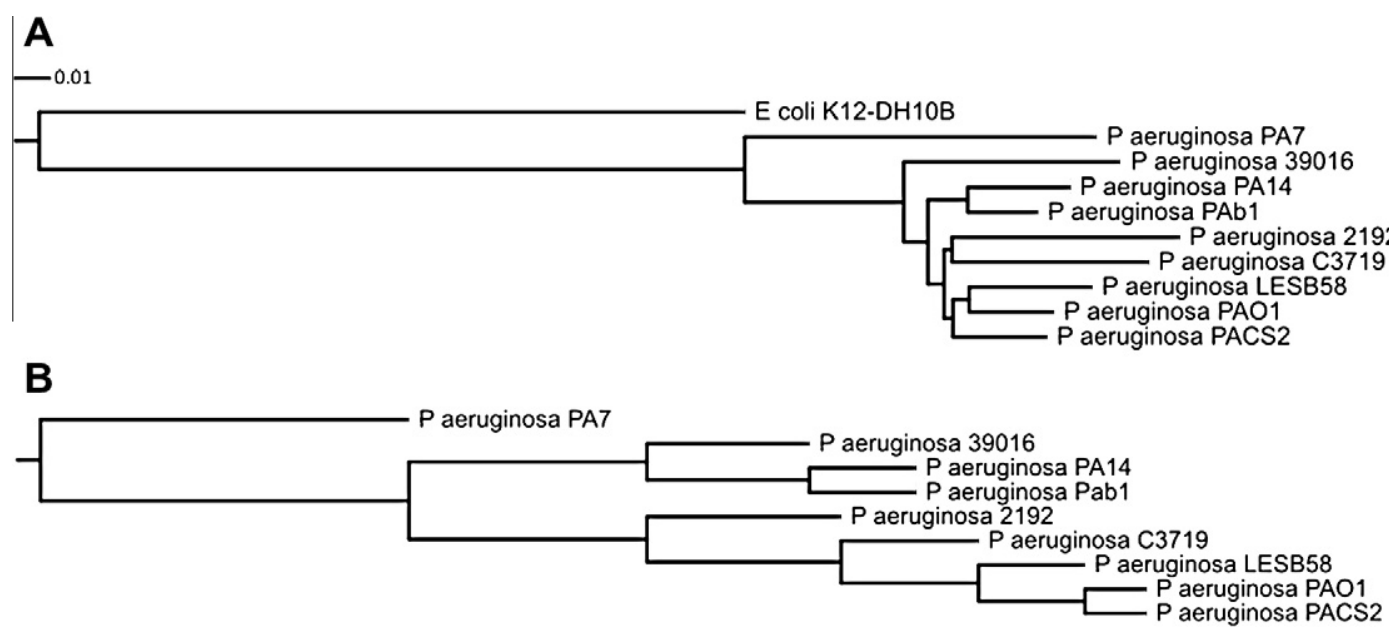

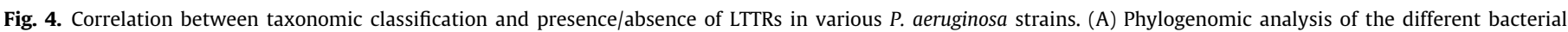

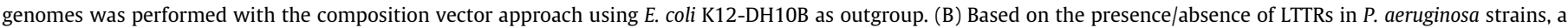
dendrogram was constructed with the Neighbour-Joining method using Euclidean distance.

the strain-specific LTTRs, PA39016_000120006 was found to have a very high genomic dissimilarity value (82.93) with a GC content of $40.2 \%$ which is more than $20 \%$ lower than the 39016 genome average. Although the function of this LTTR remains to be elucidated, the upstream low \%GC region encodes several genes with functions related to antibiotic efflux and catalase activity. Interestingly, BphR, CynR and PA14_32700 are found together in cluster $\mathbf{V}$, while PA14_03530 and PA14_46330 are in cluster III in the phylogenetic tree, perhaps an indication that they diverged from common acquisitions. This, together with the lack of evidence for acquisition in the other LTTR clusters, suggests a low frequency of acquisition. In light of the significant genomic plasticity of $P$. aeruginosa, it is surprising that only $4 \%$ of the entire LTTR repertoire across all strains show evidence of acquisition. To investigate the possibility that acquisition events occurred early in the evolutionary history of this species, BLAST analysis of the individual $P$. aeruginosa LTTRs was undertaken (excluding those that are widely distributed among the Pseudomonas genus). Several potential acquisition events were identified based on conservation of gene order, sequence identity and phylogenetic analysis, suggesting transfer events involving P. aeruginosa and Yersinia (PA14_22550) and Burkholderia (PA14_12140) may have occurred (Table S3 and Fig. S4). However, more detailed analysis will be required to characterise any potential HGT events and to fully detail their impact on the LTTR repertoire in $P$. aeruginosa.

A recent study on the GbuR, GpuR, and BauR LTTRs in $P$. aeruginosa has proposed that they are derived from a common ancestor, possibly through gene duplication and subsequent divergent evolution (Nakada and Itoh, 2005). To investigate the degree to which positive selection and divergent evolution may underpin the diversity of LTTRs in P. aeruginosa, the McDonald-Kreitman test was performed on a subset of LTTR proteins encoded within the Pseudomonas genus. Using the DnaSP software programme (Rozas et al., 2003), MK tests were performed on pooled LTTR sequences from eight $P$. aeruginosa strains (PA14, PAO1, LES, 2192, C3719, PA7, 39016 and PACS2) using $P$. fluorescens SBW25 as an outgroup to assess positive selection. Four conserved housekeeping genes (gyrB, recA, rpoD and $d n a K)$ were also selected for this analysis. Interestingly, seven of the twelve proteins chosen for analysis demonstrated significant evidence for positive selection (Table 2), while five proteins did not exhibit a statistically significant result, indicating neutral selection. Therefore, while divergence may certainly go some way towards explaining the evolutionary path of LTTR proteins in $P$. aeruginosa, it would not appear to be the only mechanism through which this large repertoire has emerged. Although we have found no study focusing specifically on LTTR evolution

Table 2

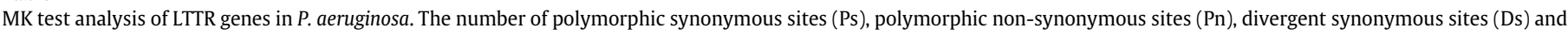
divergent non-synonymous sites (Dn) are presented.

\begin{tabular}{|c|c|c|c|c|c|c|c|c|c|}
\hline Locus ID & Gene ID & Cluster protein no. & Ps & Pn & Ds & $D n$ & Neutrality index (NI) & $p$-Value & $G$ value \\
\hline & gyrB & & 34 & 101 & 110 & 275 & 1.188 & 0.503 & 0.58 \\
\hline & dnaK & & 49 & 6 & 229 & 97 & 0.289 & 0.003 & 0.003 \\
\hline & recA & & 9 & 24 & 56 & 94 & 1.589 & 0.319 & 0.365 \\
\hline & $r p o D$ & & 28 & 29 & 104 & 94 & 0.555 & 0.052 & 0.062 \\
\hline PA14_20130 & & 1 & 14 & 9 & 97 & 84 & 0.742 & 0.657 & 0.66 \\
\hline PA14_41870 & CysB & 2 & 59 & 1 & 85 & 34 & 0.042 & 0 & 0 \\
\hline PA14_17900 & metR & 3 & 57 & 16 & 68 & 47 & 0.406 & 0.007 & 0.01 \\
\hline PA14_70560 & & 4 & 30 & 0 & 92 & 40 & 0 & 0 & na \\
\hline PA14_22470 & & 5 & 43 & 7 & 92 & 74 & 0.202 & 0 & 0 \\
\hline PA14_17380 & & 6 & 23 & 34 & 74 & 115 & 0.951 & 0.871 & 1 \\
\hline PA14_71640 & & 7 & 25 & 1 & 124 & 63 & 0.079 & 0.001 & 0.001 \\
\hline PA14_31560 & & 8 & 32 & 6 & 66 & 50 & 0.248 & 0.002 & 0.003 \\
\hline PA14_23730 & & 9 & 34 & 19 & 81 & 84 & 0.539 & 0.06 & 0.078 \\
\hline PA14_02650 & & 11 & 35 & 29 & 61 & 92 & 0.549 & 0.052 & 0.064 \\
\hline PA14_46060 & & 22 & 8 & 46 & 49 & 106 & 2.658 & 0.021 & 0.022 \\
\hline PA14_52920 & & 51 & 18 & 18 & 78 & 104 & 0.75 & 0.466 & 0.546 \\
\hline
\end{tabular}


in any organism, studies on the degree to which HGT and gene duplication events contribute to prokaryotic evolution tend to be conflicting. Most studies consider adaptive gene duplication to be the leading cause of the expansion of protein families in prokaryotes (for example, Wagner, 2008) however other studies indicate that HGT is the leading cause of protein family evolution and expansion (Price et al., 2008). Our findings indicate that the majority of LTTRs have arisen as a result of duplications or a series of convergent evolutionary events, while there would appear to be little evidence for domain shuffling based on the conservation of the cluster patterns within the DBD and CBD trees. However, we must also consider the possibility that HGT events may have occurred early in the evolutionary history of the species and this requires further investigation.

\subsection{Functional and transcriptional correlation within LTTR clusters}

To investigate the expression of $P$. aeruginosa LTTRs we generated databases from available transcriptome analyses in $P$. aeruginosa. The LTTR genes were selected and compared across a range of stimuli and mutant backgrounds to assess if any cluster patterns existed regarding their regulation. In light of the cluster conservation observed in the phylogenetic analysis, and the fact that LTTR activity and expression is controlled by small molecules binding to the C-terminal domain, the possibility that LTTRs present in the same cluster may exhibit similar expression profiles in response to broad spectrum environmental and host signals was considered. However, cluster analysis of the expression profiles revealed no correlation between sequence clusters and expression clusters suggesting that each LTTR is insulated by activation in response to diverse signals or conditions (Table S5). Notwithstanding this, a striking feature of the transcriptome database analysis was the number of LTTRs that were upregulated in response to oxidative stress. In total, 39 LTTRs were induced greater than 2-fold in response to $\mathrm{H}_{2} \mathrm{O}_{2}$ in minimal media, while only 2 LTTRs (PA1853 and PA1422) were downregulated (Palma et al., 2004). As the availability of transcriptomic datasets continues to grow it will be interesting to see if other signals are capable of global activation of LTTRs.

Interestingly, reports citing the capacity for LTTRs to bind the promoters of other transcriptional regulators have increased in frequency in recent years. Both CysB (sulphur metabolism) and OxyR (oxidative stress) have recently been shown to bind the pvdS promoter, a central regulator of iron homeostasis (Imperi et al., 2010; Wei et al., 2012). We have also found that PA2206, a non-classical LTTR required for an efficient oxidative stress response and zebrafish lethality, also bound the $p v d S$ promoter and activated $p v d S$ expression $>5$-fold upon overexpression (Reen et al., in press). What is intriguing is the fact that all three LTTRs are contained within cluster $\mathbf{V}$, suggesting that their ability to bind a common promoter sequence may arise as a consequence of conserved motifs within their respective protein structure. However, the apparent lack of correlation between the OxyR and PA2206 regulons would suggest that while cross talk may occur, significant insulation has been maintained between these regulatory systems. In addition, cross-interaction studies in E. coli have shown that LTTRs may interact to form hetero-dimeric complexes (Knapp and $\mathrm{Hu}$, 2010). However, there was no evidence that this correlated with evolutionary distance (Knapp and $\mathrm{Hu}, 2010$ ). Consistent with this assertion, while CynR and OxyR were shown to interact and are present in cluster $\mathbf{V}$, no interaction was observed between CynR and either CysB or MetR, both of which are also found in cluster V. Therefore, while certain residual traits may remain from common ancestral proteins, it is clear that each LTTR possesses distinct properties related to signal recognition and oligomerisation.

\section{Conclusion}

In terms of evolution, prokaryotic transcriptional regulatory networks are believed to be very flexible, and quickly adapt to environmental changes by modifying specific individual interactions to ultimately settle on the most advantageous organism specific design (Madan Babu et al., 2006). The evolution and expansion of transcriptional regulator families, such as LTTRs possibly arises due to a greater metabolic diversity, environmental changes, duplication and horizontal gene or operon transfer (Andersson and Hughes, 2009). The high degree of sequence identity between LTTRs indicates that they evolved from a distant common ancestor, a hypothesis supported by studies which show them to have a similar tertiary structure (Lochowska et al., 2004). Madan Babu et al. (2006) described how transcriptional regulatory networks can be viewed at three different levels with different evolutionary dynamics at each level. These include evolution of target genes, evolution at the motif (local structure) and global levels, and evolution leading to expansion of protein families (Madan Babu et al., 2006). Evolution of transcription factors was found to be rapid, generally occurring independently of target genes, with the most common factor being the evolution of new members to respond to new signals in the environment. Similar regulatory interactions were found to be conserved in bacteria that have a similar lifestyle. For example, an expansion of the LTTR family (noted for their recognition of diverse signals as coinducers) of transcription factors was noted in free-living phylogenetically distant bacteria, including Proteobacteria (which includes Pseudomonas), Streptomyces and B. subtilis but no homologues were found in any of their close relatives which are known to be obligate pathogens. The LTTR repertoire among the free-living bacteria was therefore postulated to be correlated with the need to respond to similar environmental signals or metabolites (Babu and Aravind, 2006). The analysis presented in this study suggests that distinct evolutionary patterns can be attributed to the large repertoire of LTTRs (which remains largely uncharacterised) encoded in $P$. aeruginosa. The available evidence suggests that the majority of LTTRs have arisen as a result of duplications or a series of convergent evolutionary events, while there would appear to be little evidence for domain shuffling. In addition, evidence for recent horizontal gene transfer events was limited, although acquisition from species with similar nucleotide compositions must be considered. Deciphering the functional relationships that exist among this important class of transcriptional regulator will be crucial to our understanding of the molecular networks that underpin microbial adaptation and infection. The cluster analysis and phylogenetic arrangements presented in this study will provide an important platform for future functional studies.

\section{Acknowledgments}

This research was supported in part by Grants awarded to FOG by the European Commission (FP7-KBBE-2012-6, CP-TP-312184; FP7-KBBE-2012-6, 311975; OCEAN.2011-2, 287589; MTKD-CT2006-042062, 036314), Science Foundation Ireland (07/IN.1/ B948; 08/RFP/GEN1295; 08/RFP/GEN1319; 09/RFP/BMT2350), the Department of Agriculture and Food (DAF RSF 06 321; DAF RSF 06 377; FIRM 08/RDC/629), the Irish Research Council for Science, Engineering and Technology (RS/2010/2413; 05/EDIV/FP107), the Health Research Board (RP/2006/271; RP/2007/290; HRA/2009/ 146), the Environmental Protection Agency (EPA2006-PhD-S-21; EPA2008-PhD-S-2), the Marine Institute (Beaufort award C2CRA 2007/082) and the Higher Education Authority of Ireland (PRTLI3; PRTLI4). 


\section{Appendix A. Supplementary material}

Supplementary data associated with this article can be found, in the online version, at http://dx.doi.org/10.1016/j.ympev.2012. 12.014 .

\section{References}

Abbott, J.C., Aanensen, D.M., Rutherford, K., Butcher, S., Spratt, B.G., 2005. WebACT an online companion for the Artemis comparison tool. Bioinformatics 21, 36653666.

Andersson, D.I., Hughes, D., 2009. Gene amplification and adaptive evolution in bacteria. Annu. Rev. Genet. 43, 167-195.

Anisimova, M., Gascuel, O., 2006. Approximate likelihood-ratio test for branches: fast, accurate, and powerful alternative. Syst. Biol. 55, 539-552.

Babu, M.M., Aravind, L., 2006. Adaptive evolution by optimizing expression levels in different environments. Trends Microbiol. 14, 11-14.

Beck, C.F., Warren, R.A., 1988. Divergent promoters, a common form of gene organization. Microbiol. Rev. 52, 318-326.

Bennasar, A., Mulet, M., Lalucat, J., Garcia-Valdes, E., 2010. PseudoMLSA: a database for multigenic sequence analysis of Pseudomonas species. BMC Microbiol. 10, 118.

Deziel, E., Gopalan, S., Tampakaki, A.P., Lepine, F., Padfield, K.E., Saucier, M., Xiao, G., Rahme, L.G., 2005. The contribution of MvfR to Pseudomonas aeruginosa pathogenesis and quorum sensing circuitry regulation: multiple quorum sensing-regulated genes are modulated without affecting lasRI, rhlRI or the production of N-acyl-L-homoserine lactones. Mol. Microbiol. 55, 998-1014.

Everett, M., Walsh, T., Guay, G., Bennett, P., 1995. GcvA, a LysR-type transcriptiona regulator protein, activates expression of the cloned Citrobacter freundii ampC beta-lactamase gene in Escherichia coli: cross-talk between DNA-binding proteins. Microbiology 141 (Pt 2), 419-430.

Ferrell, E., Carty, N.L., Colmer-Hamood, J.A., Hamood, A.N., West, S.E., 2008 Regulation of Pseudomonas aeruginosa ptxR by Vfr. Microbiology 154, 431-439.

Gogarten, J.P., Doolittle, W.F., Lawrence, J.G., 2002. Prokaryotic evolution in light of gene transfer. Mol. Biol. Evol. 19, 2226-2238.

Govan, J.R., Deretic, V., 1996. Microbial pathogenesis in cystic fibrosis: mucoid Pseudomonas aeruginosa and Burkholderia cepacia. Microbiol. Rev. 60, 539-574.

Guindon, S., Gascuel, O., 2003. A simple, fast, and accurate algorithm to estimate large phylogenies by maximum likelihood. Syst. Biol. 52, 696-704.

Henikoff, S., Haughn, G.W., Calvo, J.M., Wallace, J.C., 1988. A large family of bacterial activator proteins. Proc. Natl. Acad. Sci. USA 85, 6602-6606.

Imperi, F., Tiburzi, F., Fimia, G.M., Visca, P., 2010. Transcriptional control of the pvdS iron starvation sigma factor gene by the master regulator of sulfur metabolism CysB in Pseudomonas aeruginosa. Environ. Microbiol. 12, 1630-1642.

Katoh, K., Toh, H., 2008. Recent developments in the MAFFT multiple sequence alignment program. Briefings Bioinformatics 9, 286-298.

Knapp, G.S., Hu, J.C., 2010. Specificity of the E. coli LysR-type transcriptional regulators. PLoS One 5, e15189.

Krushkal, J., Qu, Y., Lovley, D.R., Adkins, R.M., 2012. Phylogenetic classification of diverse LysR-type transcriptional regulators of a model prokaryote Geobacter sulfurreducens. J. Mol. Evol. 74, 187-205.

Letunic, I., Bork, P., 2011. Interactive Tree Of Life v2: online annotation and display of phylogenetic trees made easy. Nucl. Acids Res. 39, W475-W478.

Li, Q., Xu, Z., Hao, B., 2010. Composition vector approach to whole-genome-based prokaryotic phylogeny: success and foundations. J. Biotechnol. 149, 115-119.

Lochowska, A., Iwanicka-Nowicka, R., Zaim, J., Witkowska-Zimny, M., Bolewska, K. Hryniewicz, M.M., 2004. Identification of activating region (AR) of Escherichia coli LysR-type transcription factor CysB and CysB contact site on RNA polymerase alpha subunit at the cysP promoter. Mol. Microbiol. 53, 791-806.

Lozada-Chavez, I., Angarica, V.E., Collado-Vides, J., Contreras-Moreira, B., 2008. The role of DNA-binding specificity in the evolution of bacterial regulatory networks. J. Mol. Biol. 379, 627-643.

Madan Babu, M., Teichmann, S.A., Aravind, L., 2006. Evolutionary dynamics of prokaryotic transcriptional regulatory networks. J. Mol. Biol. 358, 614-633.

Maddocks, S.E., Oyston, P.C., 2008. Structure and function of the LysR-type transcriptional regulator (LTTR) family proteins. Microbiology 154, 3609-3623.

Markowitz, V.M., Chen, I.-M.A., Palaniappan, K., Chu, K., Szeto, E., Grechkin, Y. Ratner, A., Jacob, B., Huang, J., Williams, P., Huntemann, M., Anderson, I., Mavromatis, K., Ivanova, N.N., Kyrpides, N.C., 2012. IMG: the integrated microbial genomes database and comparative analysis system. Nucl. Acids Res. 40, D115-D122.
Monferrer, D., Tralau, T., Kertesz, M.A., Dix, I., Sola, M., Uson, I., 2010. Structural studies on the full-length LysR-type regulator TsaR from Comamonas testosteroni T-2 reveal a novel open conformation of the tetrameric LTTR fold. Mol. Microbiol. 75, 1199-1214.

Nakada, Y. Itoh, Y., 2005. Pseudomonas aeruginosa PAO1 genes for 3guanidinopropionate and 4-guanidinobutyrate utilization may be derived from a common ancestor. Microbiology 151, 4055-4062.

Ochman, H., Lawrence, J.G., Groisman, E.A., 2000. Lateral gene transfer and the nature of bacterial innovation. Nature 405, 299-304.

Pallen, M.J., Wren, B.W., 2007. Bacterial pathogenomics. Nature 449, 835-842.

Palma, M., DeLuca, D., Worgall, S., Quadri, L.E., 2004. Transcriptome analysis of the response of Pseudomonas aeruginosa to hydrogen peroxide. J. Bacteriol. 186, 248-252.

Price, M.N., Dehal, P.S., Arkin, A.P., 2008. Horizontal gene transfer and the evolution of transcriptional regulation in Escherichia coli. Genome Biol. 9, R4.

Qi, J., Wang, B., Hao, B.-I., 2004. Whole proteome prokaryote phylogeny without sequence alignment: a $\langle i\rangle\langle\langle i\rangle$-string composition approach. J. Mol. Evol. 58, 111.

Redondo-Nieto, M., Barret, M., Morrisey, J.P., Germaine, K. Martínez-Granero, F. Barahona, E., Navazo, A., Sánchez-Contreras, M., Moynihan, J.A., Giddens, S.R., Coppoolse, E.R., Muriel, C., Stiekema, W.J., Rainey, P.B., Dowling, D., O’Gara, F., Martín, M., Rivilla, R., 2012. Genome sequence of the biocontrol strain Pseudomonas fluorescens F113. J. Bacteriol. 194, 1273-1274.

Reen, F.J., Haynes, J.M., Mooij, M.J., O'Gara, F., in press. A non-classical LysR-type transcriptional regulator PA2206 is required for an effective oxidative stress response in Pseudomonas aeruginosa. PLoS One

Roy, P.H., Tetu, S.G., Larouche, A., Elbourne, L., Tremblay, S., Ren, Q., Dodson, R., Harkins, D., Shay, R., Watkins, K., Mahamoud, Y., Paulsen, I.T., 2010. Complete genome sequence of the multiresistant taxonomic outlier Pseudomonas aeruginosa PA7. PLoS One 5, e8842.

Rozas, J., Sanchez-DelBarrio, J.C., Messeguer, X., Rozas, R., 2003. DnaSP, DNA polymorphism analyses by the coalescent and other methods. Bioinformatics 19, 2496-2497.

Sainsbury, S., Lane, L.A., Ren, J., Gilbert, R.J., Saunders, N.J., Robinson, C.V., Stuart, D.I., Owens, R.J., 2009. The structure of CrgA from Neisseria meningitidis reveals a new octameric assembly state for LysR transcriptional regulators. Nucl. Acids Res. 37, 4545-4558.

Schell, M.A., 1993. Molecular biology of the LysR family of transcriptional regulators. Annu. Rev. Microbiol. 47, 597-626.

Schlaman, H.R., Okker, R.J., Lugtenberg, B.J., 1992. Regulation of nodulation gene expression by NodD in rhizobia. J. Bacteriol. 174, 5177-5182.

Tian, Z.X., Fargier, E., Mac Aogain, M., Adams, C., Wang, Y.P., O’Gara, F., 2009a. Transcriptome profiling defines a novel regulon modulated by the LysR-type transcriptional regulator MexT in Pseudomonas aeruginosa. Nucl. Acids Res. 37 7546-7559.

Tian, Z.X., Mac Aogain, M., O'Connor, H.F., Fargier, E., Mooij, M.J., Adams, C., Wang, Y.P., O'Gara, F., 2009b. MexT modulates virulence determinants in Pseudomonas aeruginosa independent of the MexEF-OprN efflux pump. Microb. Pathog. 47, 237-241.

Tropel, D., van der Meer, J.R., 2004. Bacterial transcriptional regulators for degradation pathways of aromatic compounds. Microbiol. Mol. Biol. Rev. 68, 474-500.

Turner, K.H., Vallet-Gely, I., Dove, S.L., 2009. Epigenetic control of virulence gene expression in Pseudomonas aeruginosa by a LysR-type transcription regulator. PLoS Genet. 5, e1000779.

van Passel, M.W., Luyf, A.C., van Kampen, A.H., Bart, A., van der Ende, A., 2005. Deltarho-web, an online tool to assess composition similarity of individual nucleic acid sequences. Bioinformatics 21, 3053-3055.

Wagner, A., 2008. Gene duplications, robustness and evolutionary innovations. Bioessays 30, 367-373.

Wei, Q., Minh, P.N., Dotsch, A., Hildebrand, F, Panmanee, W., Elfarash, A., Schulz, S. Plaisance, S., Charlier, D., Hassett, D., Haussler, S., Cornelis, P., 2012. Global regulation of gene expression by OxyR in an important human opportunistic pathogen. Nucl. Acids Res. 40, 4320-4333.

Whelan, S., Goldman, N., 2001. A general empirical model of protein evolution derived from multiple protein families using a maximum-likelihood approach. Mol. Biol. Evol. 18, 691-699.

Winsor, G.L., Lam, D.K., Fleming, L., Lo, R., Whiteside, M.D., Yu, N.Y., Hancock, R.E., Brinkman, F.S., 2011. Pseudomonas genome database: improved comparative analysis and population genomics capability for Pseudomonas genomes. Nucl. Acids Res. 39, D596-600.

Yan, A., Wang, X., Zhang, X., Xu, Y., 2007. LysR family factor PltR positively regulates pyoluteorin production in a pathway-specific manner in Pseudomonas sp. M18. Sci. China C Life Sci. 50, 518-524. 